\title{
AN APPROACH TO BOYLE'S CONJECTURE
}

\author{
by DINH VAN HUYNH and S. TARIQ RIZVI
}

(Received 31st May 1995)

\begin{abstract}
A ring $R$ is called a right QI-ring if every quasi-injective right $R$-module is injective. The well-known Boyle's Conjecture states that any right QI-ring is right hereditary. In this paper we show that if every continuous right module over a ring $R$ is injective, then $R$ is semisimple artinian. In fact, if every singular continuous right $R$-module satisfying the restricted semisimple condition is injective, then $R$ is right hereditary. Moreover, in this case, every singular right $R$-module is injective.
\end{abstract}

1991 Mathematics subject classification: Primary 16A50, 16E60; Secondary 16D70.

\section{Introduction}

Rings for which every quasi-injective right module is injective were introduced as right QI-rings by Boyle $([1,2])$ and were studied by many authors (see for example, [3, $10,12,13])$. In Byrd [4], these rings were called right QII-rings.

A ring $R$ is called right hereditary if every right ideal of $R$ is projective, or equivalently, if every submodule (resp., factor module) of any projective (resp., injective) right $\boldsymbol{R}$-module is projective (resp., injective). If every simple (resp., singular) right $R$-module is injective, then $R$ is said to be a right V- (resp., SI-) ring. SI-rings were introduced and investigated by Goodearl in [11]. In particular, any right SI-ring is right hereditary.

It was shown by Boyle [1] that (two-sided) noetherian hereditary V-rings are QIrings. An example of Cozzens [6] shows the existence of a non-artinian QI-ring which is also an SI-domain. All known examples of QI-rings are hereditary and two-sided QI. Boyle has conjectured that:

\section{Right $Q I$-rings are right hereditary}

(cf. Cozzens-Faith [7, p. 116] and Faith [10]). It is also unknown whether or not a right QI-ring is left QI. This question is unanswered even if we assume, in addition, that the right QI-ring is right SI.

In this paper, instead of QI-rings, we study rings for which all continuous modules are injective and show that such a ring is semisimple artinian. Moreover, if we require the injectivity only for the singular continuous right $R$-modules whose factor modules by essential submodules are semisimple, then $R$ is right SI, in particular, $R$ is right 
hereditary. These results may provide an alternative approach to an answer of Boyle's Conjecture.

\section{The results}

Throughout, we consider associative rings with identity and all modules are unitary modules. For a module $M$ we denote by $Z(M)$, $S o c(M)$ and $E(M)$ the singular submodule, the socle and the injective hull of $M$, respectively.

For a given module $M$ we consider the following conditions:

$\left(C_{1}\right)$ Every submodule of $M$ is essential in a direct summand.

$\left(C_{2}\right)$ Every submodule of $M$ isomorphic to a direct summand of $M$ is itself a direct summand.

$\left(C_{3}\right)$ If $H$ and $K$ are direct summands of $M$ with $H \cap K=0$, then $H \oplus K$ is a direct summand.

A module is called continuous if it satisfies conditions $\left(C_{1}\right)$ and $\left(C_{2}\right)$, quasi-continuous if it satisfies $\left(C_{1}\right)$ and $\left(C_{3}\right)$, and extending (or CS) if it satisfies $\left(C_{1}\right)$ only.

We refer to [8] and [14] for details.

Every quasi-injective module is continuous and the hierarchy is as follows

$$
\text { injective } \Rightarrow \text { quasi-injective } \Rightarrow \text { continuous } \Rightarrow \text { quasi-continuous } \Rightarrow \text { extending. }
$$

In general, these classes of modules are distinct. In this paper, among other results, we show that, over a ring $R$, these classes of modules coincide if and only if $R$ is a semisimple artinian ring (Corollary 2)

We start with the following useful lemma which provides the existence of continuous submodules in an indecomposable quasi-injective module.

Lemma 1. Let $M$ be a quasi-injective right $R$-module. If $H$ is an essential submodule of $M$ such that $M / H$ is noetherian, then every monomorphism of $H$ into $H$ is an isomorphism. In addition, if $M$ is indecomposable, then $H$ is a continuous module.

Proof. The fact that every noetherian module cannot be isomorphic to a proper homomorphic image of itself, is known, but we include a proof for the sake of completeness. Let $A$ be a noetherian module. Then there is a submodule $B$ of $A$ which is maximal with respect to the condition that $A \cong A / B$. Let $\varphi$ be an isomorphism $A \rightarrow A / B$. Then we have $A / B \cong(A / B) / \varphi(B)$. Hence $A \cong(A / B) / \varphi(B)$. If $B$ is nonzero, then $\varphi(B)$ is nonzero in $A / B$. This would imply the existence of a submodule $C$ of $A$ containing $B$ properly and $A \cong A / C$, a contradiction to the maximality of $B$. Hence $B=0$.

Now let $M$ be a quasi-injective right $R$-module and $H$ be an essential submodule of $M$ such that $M / H$ is noetherian. Let $f$ be a monomorphism of $H$ into $H$. Assume that $f(H) \neq H$. Since $M$ is quasi-injective and $H$ is essential in $M, f$ can be extended to an automorphism $f^{\prime}$ of $M$. We obviously have 


$$
M / H \cong f^{\prime}(M) / f^{\prime}(H)=M / f(H) .
$$

But this is a contradiction since $M / f(H)$ is then noetherian and $M / H$ is a proper homomorphic image of $M / f(H)$. Thus $f(H)=H$. Now if $M$ is indecomposable, then $H$ is uniform, and so it follows that $H$ is continuous, completing the proof.

From Lemma 1, it follows for example, that if $R$ is a ring such that $E\left(R_{R}\right) / R$ is noetherian, then $R$ is the classical right quotient ring of itself: Since, for any regular element $c \in R$, the mapping $r \rightarrow c r$ for all $r \in R$ is a monomorphism hence an automorphism of $R_{R}$. Thus $R=c R$. Furthermore, if $R$ is a right noetherian right Vring, then any indecomposable quasi-injective right $R$-module is either simple or it contains infinitely many non-zero continuous proper submodules, e.g. all of its maximal submodules.

Now, let $R$ be a ring such that every continuous right $R$-module is injective. Then $R$ is a right QI-ring and hence $R$ is a right noetherian and right V-ring by [1]. There are finitely many independent uniform right ideals of $R$, say $U_{1}, \ldots, U_{n}$, such that the direct sum $U_{1} \oplus \cdots \oplus U_{n}$ is an essential right ideal of $R$. Each $E\left(U_{i}\right)$ has a maximal submodule $M_{i}$ which is continuous by Lemma 1 . By our hypothesis, $M_{i}$ is injective. Thus $M_{i}=0$, proving that each $U_{i}$ is simple and injective. Hence we have proved the following consequence of Lemma 1:

Corollary 2. A ring $R$ is semisimple artinian if and only if every continuous right $R$-module is injective.

Since semisimple artinian rings are characterized by requiring all modules to be injective, Corollary 2 shows that the concept of continuity is, in some sense, not "close" to that of injectivity.

On the other hand, if all finitely generated right $R$-modules are (quasi-) continuous, and $C$ is a cyclic right $R$-module, then $R_{R} \oplus C$ is (quasi-)continuous, hence $C$ is injective, implying that $R$ is semisimple artinian. This indicates that continuity and injectivity are "close" to each other in some ways.

It would be interesting to know about the structure of rings whose extending modules are continuous or whose quasi-continuous modules are quasi-injective.

By [3, Theorem 8] every non-singular quasi-injective module over a semiprime right Goldie ring is injective. Moreover, by [15, Corollary 5], every continuous module over a commutative noetherian ring is quasi-injective. Hence, every non-singular continuous module over a commutative noetherian semiprime ring is injective. From this and Corollary 2, not all singular continuous modules over such a ring are necessarily injective. The ring of integers is an example which exhibits this conclusion.

For a commutative QI-ring $R,[15$, Corollary 5] provides the fact that every continuous $R$-module is injective. Hence, by Corollary $2, R$ is a direct sum of finitely many fields. This is also a consequence of [4, Proposition 2], or of the fact that a commutative V-ring is von Neumann regular. 
Further, [3, Theorem 8] together with [11, Theorem 3.11] shows that a right SIdomain $D$ is right QI. Hence by Corollary 2 , if $D$ is not a division ring, then a nonsingular continuous right $D$-module is not necessarily quasi-injective.

A module $M$ is said to satisfy RSSC (restricted semisimple condition) if for each essential submodule $E$ of $M, M / E$ is semisimple. Every semisimple module satisfies RSSC, but the converse is not true in general.

Motivated by Corollary 2, we restricted our consideration to the case when singular continuous modules are injective, and show in Theorem 3 below that this condition characterizes precisely the right SI-rings of Goodearl [11].

Theorem 3. For a ring $R$ the following conditions are equivalent:

(a) $R$ is a right SI-ring;

(b) Every singular continuous right $R$-module is injective;

(c) Every singular continuous right $R$-module satisfying $R S S C$ is injective.

In this case $R$ is right hereditary.

Proof. (a) $\Rightarrow$ (b) $\Rightarrow$ (c) are clear.

(c) $\Rightarrow$ (a). Let $M$ be a cyclic singular right $R$-module. If we can show that $M$ is semisimple, then it follows that any singular right $R$-module is semisimple. Hence by (c), every singular right $R$-module is injective, proving (a).

First we claim that $M$ has finite uniform dimension. Assume on the contrary that $M$ has infinite uniform dimension. Then in $M$ there is an infinite direct sum of cyclic non-zero submodules $x_{i} R$ :

$$
\bigoplus x_{i} R \subseteq M
$$

Let $M_{i}$ be a maximal submodule of $x_{i} R$ and $L=\oplus M_{i}$. Then, $M / L$ contains an infinite direct sum $K$ of minimal submodules with

$$
K \cong \bigoplus\left(x_{i} R / M_{i}\right)
$$

Since $K$ is singular and semisimple (in particular, it is quasi-injective and satisfies RSSC), $K$ is injective by (c). Consequently, $K$ is a direct summand of the cyclic module $M / L$, a contradiction. Thus $M$ has finite uniform dimension, as desired.

To prove that $M$ is semisimple, let $U_{1}, \ldots, U_{m}$ be finitely many independent uniform submodules of $M$ whose direct sum is essential in $M$. First we show that each $U_{i}$ is noetherian. Assume that there is an infinite strictly ascending chain

$$
y_{1} R \subset y_{1} R+y_{2} R \subset \cdots
$$


of submodules of $U_{i}$. Since each singular simple right $R$-module is injective, we can find a submodule $H$ contained in the union of the members of this ascending chain, such that $M / H$ has an infinitely generated socle, which is injective, hence a direct summand of the cyclic module $M / H$, a contradiction. Hence each $U_{i}$ is noetherian.

Next we show that each $U_{i}$ is artinian. Assume that there is a $U_{i}$ which is not artinian. Then $U_{i}$ contains a submodule $N$ which is maximal with respect to the condition that $K=U_{i} / N$ is not artinian. Hence each factor module of $K$ by its non-zero submodule is artinian, and therefore semisimple. This means that $K$ is a uniform module with RSSC. Put $V=E(K)$. Then the sum $W$ of all cyclic submodules of $V$ satisfying RSSC is a nonzero fully invariant submodule of $V$. Moreover, $W$, also satisfies RSSC. Hence $Z(V) \cap W$ is a non-zero fully invariant submodule of $V$, and so it is a quasi-injective singular module satisfying RSSC. By (c), $Z(V) \cap W$ is injective. Thus $V=Z(V) \cap W$, proving that $V$ is singular and satisfies RSSC. Now, let $x$ be a non-zero element of $V$. Then $x R$ contains a maximal submodule, say $X$. Since $x R / X$ is a singular minimal submodule of $V / X, x R / X$ is injective and hence it splits in $V / X$. This shows that $V$ contains a maximal submodule, say $Y$. By Lemma $1, Y$ is continuous and hence $Y$ is injective by (c) because $Y$ satisfies RSSC. It follows $Y=0$, proving that $K$ is a simple module, a contradiction. Hence each $U_{i}$ is artinian. As $U_{i}$ is a $V$-module, $U_{i}$ must be simple and injective.

Thus, the direct sum $U$ of these $U_{i}$ 's is semisimple. Consequently, $U$ is injective, and so $U=M$, proving that $M$ is semisimple, as desired.

By [11, Proposition 3.3], any right SI-ring is right hereditary.

While a right QI-ring is right noetherian, a ring of Theorem 3 may have infinite right uniform dimension (see [11, Example 3.2]).

A ring $R$ is said to satisfy the restricted right (left) minimum condition if for each essential right ideal $E$ of $R, R / E$ is an artinian right (left) $R$-module. By Chatters [5], a two-sided noetherian, hereditary ring satisfies the restricted right (and hence left) minimum condition. Hence, as pointed out by Faith [10], for a two-sided QI-ring $R$, the presence of the restricted right minimum condition in $R$ is necessary for the truth of Boyle's Conjecture. In this connection, we note that a right noetherian right V-ring is right SI (and hence right hereditary) if and only if $R$ satisfies the restricted right minimum condition (cf. [11, Propositions 3.1 and 3.3]). Thus, by Theorem 3 and the known fact that a two-sided noetherian right hereditary ring is left hereditary, it follows that a two-sided QI-ring $R$ is hereditary if and only if every singular continuous right $R$-module is injective if and only if $R$ is a right SI-ring.

However, the question whether a right hereditary right QI-ring is necessarily right SI, remains open.

Recall that a ring $R$ is said to satisfy the restricted right socle condition if for each essential proper right ideal $I, R / I$ has non-zero socle.

Note that for right noetherian right V-rings, in particular for right QI-rings, the three concepts "RSSC", "restricted right minimum condition" and "restricted right socle condition" coincide.

By [10, Theorem 18], a right QI-ring $R$ with restricted right socle condition is right hereditary. This result can be extended as follows: 
Proposition 4. For a ring $R$ the following conditions are equivalent:

(i) $R$ is a right $Q I-$-ring with the restricted right socle condition;

(ii) $\operatorname{Soc}\left(R_{R}\right)$ and all singular continuous right $R$-modules are injective.

(iii) $\operatorname{Soc}\left(R_{R}\right)$ and all singular continuous right $R$-modules satisfying $R S S C$ are injective.

In this case, $R$ is right SI and hence right hereditary.

Proof. (i) $\Rightarrow$ (ii). By (i), $R$ is a right noetherian right V-ring. Hence every semisimple right $R$-module is injective. Clearly, (ii) follows from this and the restricted right socle condition. The implication (ii) $\Rightarrow$ (iii) is trivial.

(iii) $\Rightarrow$ (i). By Theorem $3, R$ is a right SI-ring. By [11, Theorem 3.11], $R$ has a ring direct decomposition:

$$
R=A \oplus B
$$

where $A / \operatorname{Soc}\left(A_{A}\right)$ is semisimple and $B$ is a semiprime right noetherian ring (with zero right (and left) socle). By (iii) we must have $A=\operatorname{Soc}\left(A_{A}\right)$, i.e. $A$ is a semisimple artinian ring. Moreover, every non-singular quasi-injective right $B$-module is injective by [3, Theorem 8]. Consequently, $R$ is a right QI-ring. Since $R$ is right SI, every singular right $R$-module is semisimple (cf. [11, Proposition 3.1]), in particular, $R$ has the restricted right socle condition, proving (i). The last statement is clear.

\section{Remarks}

In view of the conclusions in Corollary 2 and Theorem 3, the answer to Boyle's Conjecture appears likely to be in the affirmative. This, however, still remains to be seen.

Our results in Corollary 2 and Theorem 3 can be easily transferred from rings to modules $M$ over a given ring $R$, via $\sigma[M]$, the full subcategory of Mod- $R$, whose objects are submodules of $M$-generated modules (cf. [16]). The arguments to be used are similar to the ones we have presented here. In the case of Theorem 3 , if $M$ is projective in $\sigma[M]$ and every $M$-singular continuous module satisfying RSSC is $M$ injective, then every submodule of $M$ is projective in $\sigma[M]$.

Acknowledgement. This research was supported in part by a research grant from the Ohio State University-Lima, and by a grant from the Math Research Institute, Columbus. The authors gratefully acknowledge this assistance.

S. Tariq Rizvi wishes to acknowledge the partial support he received from NSF, grant number INT 9318322. 


\section{REFERENCES}

1. A. K. Boyle, Hereditary QI-rings, Trans. Amer. Math. Soc. 192 (1974), 115-120.

2. A. K. Boyle, Injectives containing no proper quasi-injective submodules, Comm. Algebra 4 (1976), 775-785.

3. A. K. Boyle and K. R. Goodearl, Rings for which certain modules are injective, Pacific J. Math. 58 [1975], 43-53.

4. K. A. BYrd, Rings whose quasi-injective modules are injective, Proc. Amer. Math. Soc. 33 (1972), 235-240.

5. A. W. Chatters, The restricted minimum condition in noetherian hereditary rings, $J$. London Math. Soc. 4 (1971), 83-87.

6. J. H. Cozzens, Homological properties of the ring of differential polynomials, Bull. Amer. Math. Soc. 76 (1970), 75-79.

7. J. H. Cozzens and C. FaITH, Simple Noetherian Rings (Cambridge University Press, 1975).

8. N. V. Dung, D. V. Huynh, P. F. Smith and R. Wisbauer, Extending Modules (Research Notes in Mathematics Series 313, Pitman, London, 1994).

9. C. FAITH, When are proper cyclics injective? Pacific J. Math. 45 (1973), 97-112.

10. C. FaItH, On hereditary rings and Boyle's Conjecture, Arch. Math. 27 (1976), 113-119.

11. K. R. GOODEARL, Singular torsion and the splitting properties (Memoirs Amer. Math. Soc. 124, 1972).

12. K. KosLer, On hereditary rings and noetherian V-rings, Pacific J. Math. 103 (1982), 467-473.

13. S. R. LOPEZ-Permouth and S. T. Rizvi, On certain classes of QI-rings, in Methods in Module Theory (Lecture Notes Vol. 140, Marcel Dekker, 1993), 227-235.

14. S. H. Mohamed and B. J. Müller, Continuous and Discrete Modules (London Math. Soc. Lecture Notes 147, Cambridge Univ. Press, 1990).

15. S. T. Rizvi, Commutative rings for which every continuous module is quasi-injective, Arch. Math. 50 (1988), 435-442.

16. R. WisBaUER, Foundations of Module and Ring Theory (Gordon and Breach, Reading, 1991).

INSTITUTE OF MATHEMATICS

P.O. BOX 631 BOHO

HANOI

VIETNAM
The OHio State University at Lima LIMA, OH 45804

U.S.A. 